VIII ${ }^{\text {èmes }}$ Journées Nationales Génie Civil - Génie Côtier, Compiègne, 7-9 septembre 2004

\title{
Erosion des fonds sableux au droit d'une digue, (site de Véran, Golfe du Lion, Méditerranée)
}

\author{
Samat Olivier $^{(\mathrm{a})}$, Lambert Adrien ${ }^{(\mathrm{a})}$, Sabatier François ${ }^{(\mathrm{a}, \mathrm{b})}$. \\ (a) CEREGE - Centre Européen de Recherche et d'Enseignement en Géosciences de l'Environnement - \\ Europôle de L'Arbois - BP 80 - 13545 Aix-en-Provence cedex 04, France \\ samat@cerege.fr, lambert@cerege.fr, sabatier@cerege.fr \\ (b) Delft University of Technology, Faculty of Civil Engineering, Hydraulic Engineering Section, Stevinweg 1, \\ 2628 CN Delft, The Netherlands.
}

\section{Résumé}

L'analyse de profils bathymétriques au droit et de part et d'autre d'une digue implantée au niveau de la position du rivage sur une côte sableuse à barres indique une augmentation des pertes sédimentaires, un approfondissement de la fosse au pied de l'ouvrage et un déplacement des barres vers le large. Une organisation longitudinale en relation avec le transit littoral dominant est aussi observée suggérant que l'analyse de l'impact d'une digue frontale doit se faire transversalement et longitudinalement.

\section{Abstract:}

Bathymetric profils survey in front of a seawall indicate : increasing of erosion, scouring and offshore bars movements in comparison to bathymetric survey without seawall. We evidence longitudinal impact of the seawall in the direction of the dominant longshore sediment transport. The erosives impact of the seawall are organized in both cross-shore and longhore directions.

\section{Introduction}

L'utilisation des digues pour protéger les côtes et limiter les inondations des tempêtes est une pratique courante en ingénierie côtière. L’impact de ces digues sur l'évolution bathymétrique, lorsqu'elles sont implantées au niveau de la position du rivage ou dans la zone de déferlement demeure encore mal connu. Certains auteurs considèrent en effet qu'une digue n'augmente pas particulièrement l'érosion de la partie sous-marine, sauf une érosion locale en pied d'ouvrage, alors que d'autres, au contraire, soulignent leur rôle négatif lié à une modification de la bathymétrie et/ou une augmentation du transport sédimentaire longshore qui amplifie les processus d'érosion ${ }^{12}$. Quoi qu'il en soit, les relevés de terrain autour des digues sont peu nombreux ce qui rend difficile toute généralisation sur l'efficacité et l'impact des digues sur les plages. Ce travail présente donc les résultats d'un programme de surveillance bathymétrique au droit d'une digue frontale sur une côte sableuse à barres en domaine microtidal, afin d'apporter des éléments nouveaux sur ces phénomènes d'érosion. Les problèmes de stabilité de cette digue ont récemment étaient dévoilés durant une forte tempête (décembre 2003) qui a provoqué son écoulement partiel.

Le site étudié se localise sur le littoral du Delta du Rhône (Mer Méditerranée, France). La plage montre une côte à barre de type « Dissipatif and Longshore-Bar-Trough » selon Wright and Short (1984) ${ }^{3}$, avec un transport longitudinal dominant dirigé vers l'Ouest (fig 1). Avant la construction en 1998 de la digue qui s'étend désormais sur 2,7km, le rivage reculait à des vitesses comprises entre -3 et $-8 \mathrm{~m}^{-a^{-1}}{ }^{4}$ principalement à cause d'une augmentation du 
gradient du transport longshore ${ }^{5}$, dont la diminution à l'Ouest se traduit par une forte sédimentation (flèche de Beauduc).

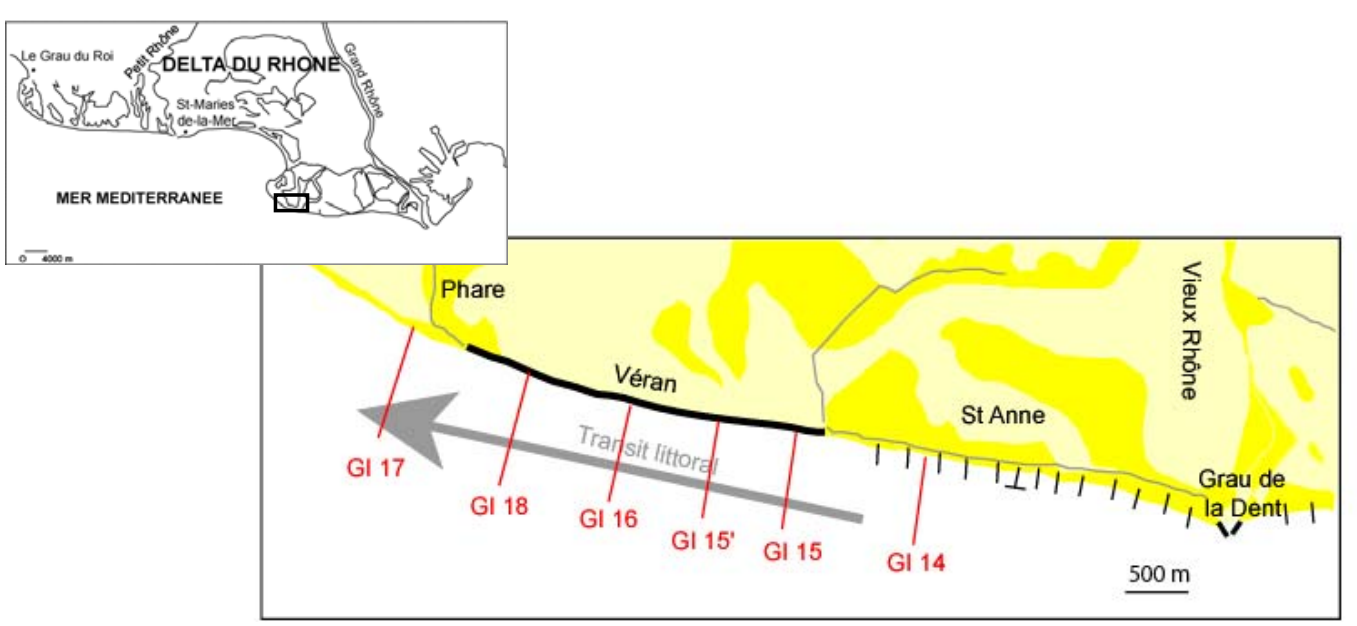

Figure 1: Localisation du site et des lignes de profil

\section{Méthodes}

Pour déterminer l'impact de la digue sur l'évolution des profils bathymétriques, nous relevons 6 lignes de profils depuis septembre 2000, à raison de quatre relevés saisonniers par an (fig1). Quatre lignes sont situées devant l'ouvrage et deux lignes de part et d'autre de celui-ci. Ces deux dernières sont censées représenter des profils «naturels » de comparaison avec celles au droit de la digue. Les profils sont réalisés du pied de la digue (pour les secteurs concernés) jusqu'à $-12 \mathrm{~m}$ de profondeur ${ }^{5}$, au moyen d'un sondeur (erreur en $\mathrm{Z}+/-0,3 \mathrm{~m}$ ) et d'un GPS différentiel embarqué sur un bateau pneumatique. Tous les profils sont recalés par rapport au NGF à partir des données marégraphiques du jour, enregistrée à moins de trois kilomètres du site.

Les données sont retranscrites graphiquement afin d'obtenir des courbes représentatives de chaque profil aux différentes dates, auxquelles plusieurs opérations sont effectuées : calcul de bilans sédimentaires moyens globaux, bilans sédimentaires et déplacement moyen des barres d'avant côte, estimation de l'affouillement au pied de l'ouvrage et de la profondeur des fosses.

\section{Résultats}

\subsection{Bilans sédimentaires des lignes de profils}

En terme de bilans sédimentaires moyen le secteur accuse une perte généralisée dont les valeurs, au niveau des lignes augmentent dans le sens de la dérive littorale dominante (fig 2). Le profil le plus à l'Est (GI14) accuse un déficit d'environ $40 \mathrm{~m}^{3} \cdot \mathrm{an}^{-1}$. Le déficit s'accélère ensuite d'Est en Ouest le long de l'ouvrage pour atteindre en GI18, la valeur maximale, de près de $250 \mathrm{~m}^{3} \cdot \mathrm{an}^{-1}$. Ce déficit diminue ensuite vers l'Ouest mais reste important avec en GI17 une érosion de l'ordre de $200 \mathrm{~m}^{3} . \mathrm{an}^{-1}$. Finalement le volume global de sable perdu extrapolé à l'ensemble du secteur compris entre GI14 et GI17 s'élève à $-545000 \mathrm{~m}^{3}$.an ${ }^{-1}$. En plus de l'analyse des bilans sédimentaires bruts, les relevés indiquent une accentuation de la variabilité des valeurs d'érosion sur les profils au droit de la digue (fig 2). 


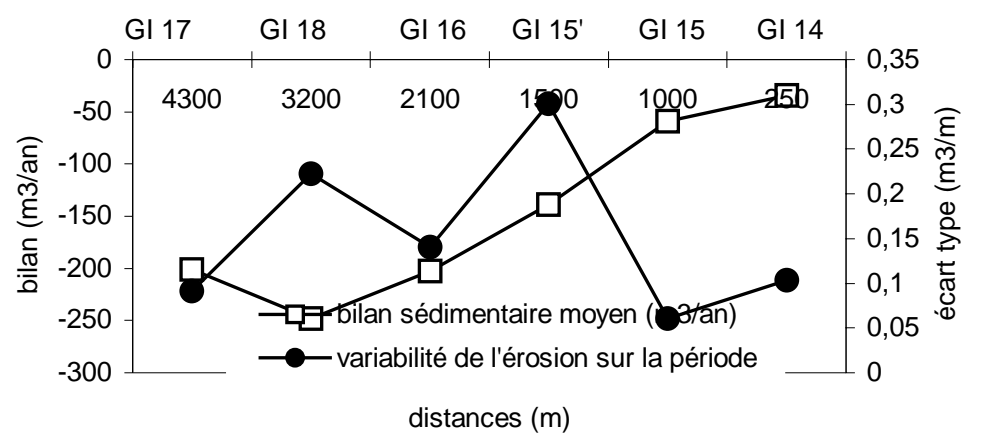

Figure 2: Variabilité et bilan sédimentaire par profil

L'extension de la zone «active» du profil, définie par la distance entre le rivage et la profondeur de fermeture s'étend sur 400m en GI14, puis augmente significativement d'Est en Ouest sur les profils devant l'ouvrage pour atteindre 1000m en GI18. la zone active diminue ensuite vers l'ouest en GI17. La profondeur de fermeture qui varie de -5 à -8 montre la même tendance. Finalement, la profondeur de fermeture se situe beaucoup plus loin en mer sur les profils au droit de la digue que sur les profils dits «naturels» (fig 3).

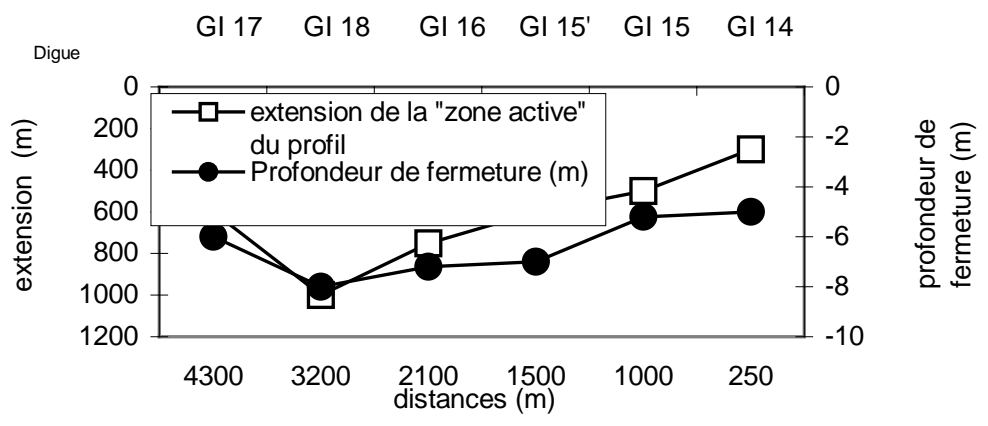

figure 3: Extension transversale de la mobilité des profils et profondeur de fermeture

\subsection{Evolution des barres}

La morphologie des profils bathymétriques évolue longitudinalement. Elle passe de deux barres relativement bien individualisées à l'Est (GI14,GI15), à trois barres au droit (GI18 et GI16) et à l'Ouest de la digue (GI17). Le bilan sédimentaire des deux premières barres (b1 et b2), sur la période, évolue de façon similaire (fig.4). On observe une perte principalement sur la partie Ouest de la digue ainsi que sur la partie médiane et un gain pour la partie Est (GI15 et GI14).
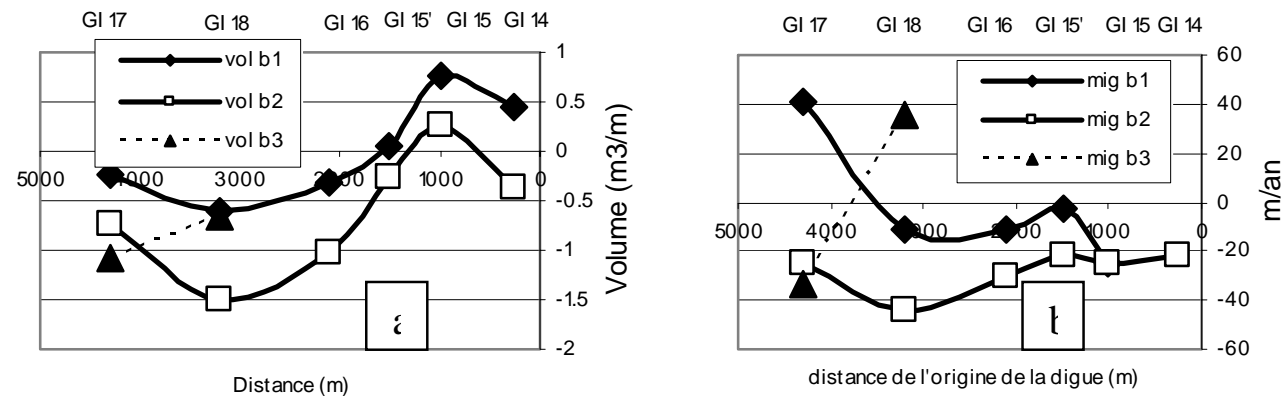

Figure 4: Evolution des barres d'avant côte (a: bilan sédimentaire; b:déplacement) 
Sur tous les profils, la barre 2 montre les plus forts taux d'érosion. Le bilan sédimentaire des deux premières barres affiche aussi une tendance (à l'érosion) dans le sens de la dérive littorale dominante. La troisième barre (b3) en GI17 et GI18 montre également des pertes importantes où elles dépassent celles de la barre 2 en GI17 (fig 4.a). La mobilité transversale des deux premières barres (b1 et b2) montre un déplacement similaire avec un recul généralisé de l'ensemble du système, accentué sur la barre 2 (sauf en GI17, fig 4.b). Les reculs les plus importants atteignent $45 \mathrm{~m} \mathrm{an}^{-1}$. La barre interne (b1), la plus proche de la digue, recule moins que la barre 2 qui est la seule à montrer une évolution spatiale Est-Ouest.

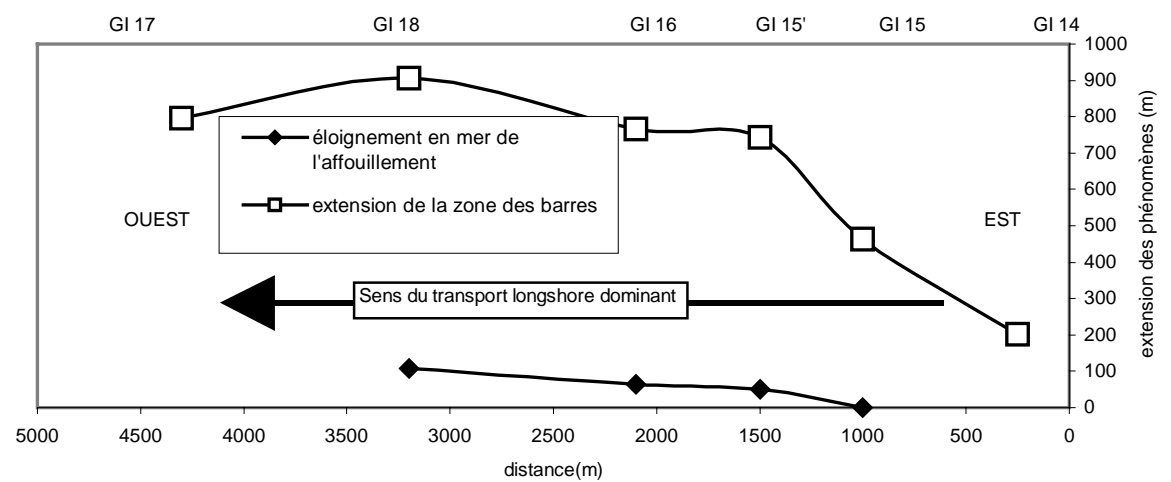

Figure 5: Répartition longitudinale de l'intensité de l'affouillement

Finalement, le recul des barres et surtout de la barre externe se traduit par un élargissement de la zone du déferlement qui s'amplifie dans le sens de la dérive dominante et surtout au droit de la digue (fig.5). Le profil « naturel » GI17 montre une migration importante de la barre interne vers la côte en relation avec un recul du rivage tandis que les barres 2 et 3 migrent aussi vers le large. (fig 4.b)

\subsection{Evolution des fosses et de l'affouillement au pied de la digue}

GI 17

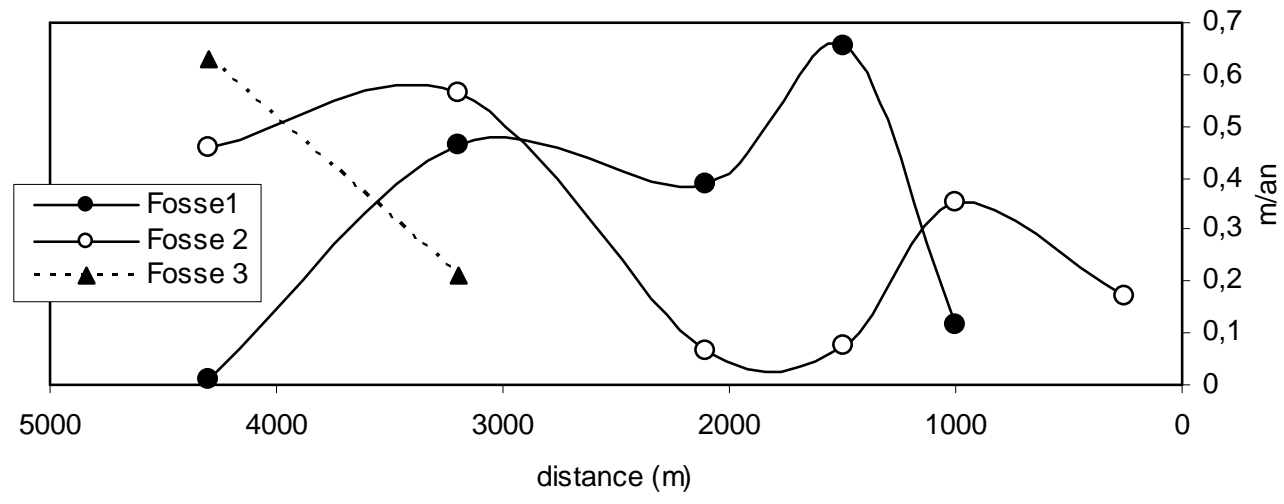

Figure 6: Approfondissement des fosses

La comparaison morphologique des profils devant la digue et «naturels » indique que la fosse interne située entre le rivage et la barre interne se localise au pied de la digue. Le phénomène essentiel est l'approfondissement des fosses sur l'ensemble des profils (fig 6). A l'Ouest, (en GI17), les plus fortes valeurs d'approfondissement se retrouvent sur les fosses les plus éloignées du rivage ( 2 et 3 ) alors que la fosse interne ne montre pas de modifications significatives. Le profil GI18 montre un approfondissement très important sur l'ensemble 
des fosses (1, 2 et 3). A l'Est, GI15 et GI14 témoignent d'une incision essentiellement marquée sur la fosse externe. Les valeurs les plus fortes se retrouvent au niveau de la fosse interne sur les profils au droit de la digue (GI18, GI16, GI15') avec des valeurs moyennes d'approfondissement de 0,45 à près de $0,7 \mathrm{~m} \mathrm{an}^{-1}$ (fig.6). Les profils encadrant la digue GI17 et GI15 (en tout début d'ouvrage), ne montrent pas d'approfondissements significatifs de leur fosse interne (les relevés de terrain n'ayant pas systématiquement permis de mesurer la zone entre le rivage et la barre interne, la fosse en GI14 n'est pas figurée sur la figure 6). La digue augmente donc l'érosion des fonds au pied de l'ouvrage. Cette érosion est perceptible par un approfondissement de la fosse interne. Enfin, si l'organisation spatiale de l'approfondissement des fosses semble complexe, une distinction longitudinale apparaît pour la fosse interne qui s'éloigne vers le large dans le sens du transit dominant (fig.5).

\section{Discussion}

L’étude met en évidence une augmentation des pertes sédimentaires, une migration vers le large des barres externes accompagnée d'une diminution de leur masse volumique et un approfondissement de la fosse au pied de l'ouvrage au droit de la digue confirmant les travaux antérieurs ${ }^{67}$ sur des plages meso et macro-tidales aux conditions de houles plus énergétiques. Cette évolution est causée par une augmentation des turbulences dues aux dynamiques réflectives liées à la présence de la digue ${ }^{8}$. La migration des barres externes vers le large ne peut être catégoriquement attribuée à la présence de la digue car ce phénomène s'observe aussi sur des côtes dépourvues d'ouvrages ${ }^{9}$, notamment sur les plages méditerranéennes du Golfe du Lion ${ }^{10,11}$. Il manque cependant à notre analyse, des relevés réalisés avant la construction de l'ouvrage pour déterminer son réel impact sur les fonds. Néanmoins, l'influence de la digue se fait très probablement ressentir à travers toute la zone du déferlement et probablement à des profondeurs supérieures car la variabilité cross-shore des profils est parfois deux fois supérieures devant la digue que sur les profils « naturels » (fig.3), illustrant une augmentation de la profondeur de fermeture.

Nos résultats montrent aussi une augmentation de l'érosion dans le sens du transit dominant, en accord avec les travaux de Sabatier ${ }^{5}$ qui expliquent l'érosion dans ce secteur par une augmentation du transport longshore Cependant, l'érosion s'amplifie au début de la digue et diminue à la fin de celle ci dans le sens du transit littoral suggérant un effet amplificateur de l'ouvrage sur les processus d'érosion. L'augmentation de l'érosion dans la direction du transit dominant était relativement peu étudiée jusqu'alors, les travaux antérieurs se concentrant surtout sur l'évolution cross-shore des profils. Nos travaux semblent néanmoins en accord avec les mesures de courantologie in-situ de Miles et al (2001) ${ }^{3}$, qui montrent aussi une augmentation longitudinale de l'érosion le long d'une digue frontale. Le profil «naturel » GI17, en aval dérive, subit probablement l'influence de la digue dont les effets longshore ont été démontrés. Le recul du rivage est ici attribuable à l'impact de la digue ${ }^{7,8,14,15}$ qui est responsable d'une érosion localisée en aval dérive. Toutefois, le trait de côte au niveau de GI17 reculait avant la construction de la digue, il est donc impossible de déterminer la part de ce recul lié à l'ouvrage, ici encore, nos conclusions souffrent de l'absence d'une analyse basée sur des relevés réalisés avant la construction de la digue.

Ces conclusions vont à l'encontre de certains travaux ${ }^{12}{ }^{13}$ qui admettent que la morphologie des fonds au droit et autour des ouvrages n'est pas fondamentalement différente. Au contraire nous montrons l'impact négatif des digues sur la bathymétrie environnante remettant en question à terme la stabilité de l'ouvrage. Cependant les réponses morphologiques et hydrodynamiques à l'implantation d'une digue dépendent largement des conditions locales : position de la digue sur le profil, tendance érosive à long terme, et le type 
de structures ${ }^{16}$. Nous soulignons de ce fait les difficultés de compréhension des phénomènes d'érosion devant une digue frontale dès lors qu'on s'intéresse à des cas d'étude et la difficulté à généraliser l’impact d’un tel ouvrage sur la bathymétrie.

\section{Conclusion}

Après trois années de suivi les variations bathymétriques montrent une organisation transversale des phénomènes sans doute en relation avec des dynamiques réflectives, (augmentation de la profondeur de fermeture, mobilité transversale du profil, et présence d'un affouillement), mais également une organisation longitudinale, (augmentation des pertes, augmentation de la mobilité générale, éloignement de l'affouillement dans le sens de la dérive littorale). Aussi les résultats indiquent que l'analyse de l'impact d'une digue frontale doit se faire transversalement et longitudinalement. Une campagne de mesures dynamiques permettra d’amener des éléments de réponses plus concrets sur ce phénomène.

\section{Bibliographie}

1.Komar P.D, Mc Dougal W.G, (1988). Coastal Erosion and engineering structures: the Oregon experience, Journal of Coastal Reseach, SI 4 77-92, Virginia.

2.Miles J.R, Russel P.E, Huntley D.A, (2001). Field measurement of sediment dynamics in front of a seawall, institute of Marine Studies University of Plymouth, journal of coastal research, 17.1, 195-206

3.Wright L.D, Short A.D, (1984) Morphodynamic variability of surf zones and beaches : a synthesis. Marine Geology, 56: 93-118

4.Sabatier F. et Suanez S. (2003) Evolution of the Rhône delta coast since the end of the $19^{\text {th }}$ century. Géomorphologie : relief, processus, environnement, 4, 283-300.

5.Sabatier F., (2001).Fonctionnement et dynamiques morpho-sédimentaires du littoral du delta du Rhône, thèse de doctorat, Université d'Aix Marseille III

6.Barnet M.R, Asce A.M, Wang H., ,(1988) Effects of a vertical seawall on profile response, $21^{\text {st }}$ Coastal engineering international conference, Malaga, vol 1-3, p 1493-1507.

7.Morton R.A, (1988) Interactions of storms, Seawalls, and Beaches of the Texas Coast, Journal of Coastal research SI 4 113-134, Virginia

8.Kraus N.C, (1988) The effects of seawalls on the beach: an extended literature review, Journal of coastal research SI 4 1-28, Virginia

9.Ruessink B.G, Kroon A.,(1995) The behaviour of a multiple bar system in a nearshore zone of Terschelling, the Netherlands, 1965-1993, Marine Geology 121, 187-197

10.Sabatier F., Provansal M. (2000) Sandbars morphology of Espiguette spit, Mediterranean Sea, France, International Workshop Sandwaves Dynamics, Lille, 23-25 march 2000, 179-187.

11.Certain, R. 2002. Morphodynamique d'une côte sableuse microtidale à barres : le golfe du lion (Languedoc-Roussillon). Thèse, Université de Perpignan, 209p.

12.Jones B.N, Basco D.R, (1997) Seawall effects on historically receding shorelines, $25^{\text {th }}$ International Conference on Coastal Engineering Sept 1996, Orlando, p1985

13.Wiegel R.L ; (2002) Seawalls, seacliffs, beachrock : what beach effects ? part1, Shore and beach vol70, $n^{\circ} 1, p$ 17-27

14.Dean R.G, (1986) Coastal Armoring: effect, principles and mitigation, in 20th International Conference Coastal engineering, vol2, p 1843-1857.

15.Griggs G.B, Tait J.F, (1988). The effects of Coastal protection structures on beaches along northern Monterey bay, California, Journal of Coastal Reseach, Virginia SI 4 93-111.

16.Plant N.G, Griggs G.B, (1992). Interactions between nearshore processes and beach morphology near a Seawall, Journal of Coastal Research 8, 1, 183-200, Florida. 